Am. J. Trop. Med. Hyg., 105(4), 2021, pp. 955-959

doi:10.4269/ajtmh.21-0436

Copyright ( 2021 by The American Society of Tropical Medicine and Hygiene

\title{
Chikungunya Fever in Southern Thailand, 2018
}

\author{
Natthaphon Nanakorn, ${ }^{1}$ Theerakamol Pengsakul, ${ }^{1}$ Kannika Bunrod, ${ }^{2}$ Suwich Thammapalo, ${ }^{3}$ Pathomporn Prikchoo, ${ }^{3}$ \\ Sompong Vongpunsawad, ${ }^{4}$ and Yong Poovorawan ${ }^{4 *}$ \\ ${ }^{1}$ Faculty of Medical Technology, Prince of Songkla University, Songkhla, Thailand; ${ }^{2}$ Langu Hospital, Satun, Thailand; ${ }^{3}$ Office of Disease Prevention \\ and Control 12, Songkhla, Thailand; ${ }^{4}$ Center of Excellence in Clinical Virology, Faculty of Medicine, Chulalongkorn University, Bangkok, Thailand
}

\begin{abstract}
Infection by the mosquito-borne chikungunya virus (CHIKV) causes acute febrile illness and debilitating arthralgia. Outbreaks are sometimes not recognized because of its clinical resemblance to the more common dengue fever ubiquitous in tropical countries. An upsurge of dengue-like illness was reported in Satun province located in southern Thailand during the rainy season in 2018. We investigated probable outbreak of CHIKV disease. We collected serum samples from 127 patients and tested for CHIKV infection based on nucleic acid and serological tests. CHIKV RNA amplified by real-time reverse-transcription polymerase chain reaction (RT-PCR) and IgM antibody against CHIKV were determined by immunochromatographic rapid test. Mosquitoes in the community were also trapped and tested for CHIKV. Conventional RT-PCR on initially positive samples was performed to obtain nucleotide sequences for subsequent phylogenetic analysis. In all, 39\% (50/127) of the samples tested positive for CHIKV RNA, IgM, or both. Of these, CHIKV RNA was identified in 17\% $(21 / 127)$ of the samples. Fourteen percent (18/127) of the samples were simultaneously positive for both IgM and IgG, which suggest recent infection. One sample tested positive for both $\mathrm{CHIKV} \mathrm{IgM} \mathrm{and} \mathrm{RNA.} \mathrm{Several} \mathrm{samples} \mathrm{from} \mathrm{Aedes} \mathrm{aegypti} \mathrm{and}$ Aedes albopictus mosquitoes were also CHIKV RNA-positive. Sequence analysis revealed that the Satun CHIKV belonged to the Indian Ocean lineage within the East/Central/South African (ECSA) clade with residues K211E and A226 in the E1 gene, and G205S and V264A in the E2 gene. The ECSA strain of CHIKV continues to evolve and possesses virulent potential despite causing prior outbreaks in the region.
\end{abstract}

\section{INTRODUCTION}

Chikungunya fever is an acute febrile illness caused by the chikungunya virus (CHIKV) infection. The virus is carried primarily by infected mosquitoes of the species Aedes albopictus and Aedes aegypti, which are found in the tropics and subtropics. Earliest documented outbreaks of chikungunya, a word derived from the Kimakonde language meaning "to become contorted," occurred in 1952 in what is now southeast Tanzania. ${ }^{1}$ Infected patients typically are presented with fever, chills, headache, myalgia, conjunctivitis, and body rash. ${ }^{2,3}$ Concurrent and prolonged symptoms after infection may include severe arthralgia. Because of the clinical manifestation similar to those caused by dengue or Zika virus, it is sometimes underrecognized and misdiagnosed. Persistent severe arthralgia is a hallmark of CHIKV infection, which often assists in the clinical diagnosis to distinguish the disease from other mosquito-borne viral infection. There is currently no treatment nor an approved vaccine in countries where CHIKV is endemic, and patients are generally offered supportive care until symptom resolution.

CHIKV is a single-strand RNA virus of positive polarity belonging to the family Togaviridae in the genus Alphavirus. ${ }^{4}$ The viral genome is $\sim 12 \mathrm{~kb}$ in length and encodes two open reading frames (ORF). ORF1 encodes four nonstructural proteins, whereas ORF2 encodes five structural proteins. The major envelope glycoproteins E1 and E2 encoded within the ORF2 are the principal components of the virion ectodomain and are involved in cell fusion and receptor binding, respectively. ${ }^{5}$ More neutralizing antibodies target E2 as it is more exposed on the viral surface compared with $\mathrm{E} 1$, and neutralization can occur by either inhibiting fusion or blocking attachment. ${ }^{6}$

*Address correspondence to Yong Poovorawan, Center of Excellence in Clinical Virology, Faculty of Medicine, Chulalongkorn University, Bangkok 10330, Thailand. E-mail: yong.p@chula.ac.th
There are three currently recognized lineages of $\mathrm{CHIKV}$, namely Eastern/Central/Southern African (ECSA), West African, and Asian, and an additional proposed Indian Ocean lineage. ${ }^{7}$ The primary mosquito vector has been Aedes aegypti, but Aedes albopictus has also supported CHIKV replication especially when the viral genome contains a specific mutation $\mathrm{A} 226 \mathrm{~V}$ in the $E 1$ gene. $^{8}$ On the $E 2$ gene, several fitness mutations have been identified, of which V264A enhances viral infectivity in A. aegypti. ${ }^{9}$

CHIKV outbreaks in Southeast Asia have been reported in Brunei, Cambodia, Indonesia, Malaysia, The Philippines, and Singapore. ${ }^{10-18}$ In Thailand, the earliest reported CHIKV was in 1958 and involved viral identification by neutralization and complement fixation tests. ${ }^{19}$ More recently, the multiprovincial CHIKV outbreak between 2008 and 2009 has affected an estimated 46,000 people. ${ }^{20,21}$ Since then, sporadic CHIKV infections are reported to the Ministry of Public Health annually.

Beginning in June 2018, which coincides with the local rainy season, physicians in Langu Hospital in Satun province of southern Thailand observed an upsurge of outpatients who presented with fever, skin rash, and joint pain. This predominantly rural region borders northern Malaysia and the Andaman Sea to the west, and whose residents mainly engage in the plantations of para rubber, oil palms, and fishing. This incidence was subsequently reported to the local office of disease prevention and control, whose staff visited local households where the patients reside. This study describes the investigation of CHIKV outbreak in southern Thailand and the molecular characterization of the CHIKV strains found in this region.

\section{MATERIALS AND METHODS}

Samples. Patients residing in the southern province of Satun who reported acute febrile illness and sought hospitalized care at Langu Hospital $(N=54)$, and symptomatic residents living in the same neighborhood as the patients $(N=73)$ were included inthisstudy. Theresidents werevisited by thestafffrom thelocal office of disease prevention and control as part of the field 
investigation. In all, a total of 127 individuals (38 males and 89 females, age range 1-74 years) were identified from June to October 2018. Demographic information and clinical symptoms, when available, were collected from the questionnaires administered by the hospital or healthcare field staff. The study was approved bythelnstitutional Review Board of the Faculty of MedicineofChulalongkornUniversity(IRBnumber378/59), and informed consent was obtained from all participants (or their parents/legal guardians if they were minors). In addition, mosquitoes were randomly collected from nearby homes where patients lived. Female $A$. aegypti and $A$. albopictus mosquitoes were specifically identified among the trapped mosquitoes. CHIKV testing was performed on 18 pools (five mosquitoes per pool) of $A$. aegypti, and three individual $A$. albopictus.

CHIKV RNA detection. Viral RNA was extracted from serum samples by using Ribospin vRD II (Geneall, Seoul, Korea). CHIKV nucleic acids were tested by using a onestep real-time reverse-transcription polymerase chain reaction (RT-PCR) (QuantiTect Probe RT-PCR kit, Qiagen, Germany) as previously described. ${ }^{22}$ Briefly, CHIKV-specific primers and probes were included in the reaction and subjected to the following conditions: reverse-transcription step at $50^{\circ} \mathrm{C}$ for 30 minutes and $95^{\circ} \mathrm{C}$ for 15 minutes, followed by 45 cycles of amplifications at $95^{\circ} \mathrm{C}$ for 15 seconds and $60^{\circ} \mathrm{C}$ for 1 minute. CHIKV Ross strain (GenBank accession number MG280943) served as a positive control.

CHIKV E1 and E2 gene amplification. CHIKV positive samples were subjected to cDNA synthesis by using ImProm II Reverse Transcription System (Promega, Madison, WI). Partial E1 gene amplification by seminested PCR was performed as previously described. ${ }^{21}$ Briefly, the first amplification reaction used forward primer DVRChkF (5'-ACCGGCCTC TACCCATTCATGT-3', nucleotides 10237-10258) and reverse primer CU3-CHIKR (5'-TCGCTRCAGCACACRGCACC-3', nucleotides 10741-10760). The second amplification reaction used a different forward primer CU1 CHIKF (5'-GCATCA GCTAAGCTCCGCGTC-3', nucleotides 10378-10398). Nucleotide positions are based on the S27-African prototype strain (GenBank accession number AF369024). After the amplicon was agarose gel-purified and subjected to Sanger sequencing, nucleotide sequences of 485 base pairs were submitted to the GenBank database under the accession numbers MK061363-MK061377. Full-length E2 gene amplification was performed using primer pairs spanning nucleotides 7968 to 10370. They were 7910F (5'-TCGAAGTCAAGCACGAA GG-3') and 8670R (5'-GTCTGTCGCTTCATTTCTGATG-3'), 8459F (5'-TGCTTGAGGACAACGTCATGAG-3') and 9240R (5'-TTTGTGATTGGTGACCGCG-3'), 9093F (5'-AGT CCGGCAACGTAAAGATCAC-3') and 9861R (5'-AAAGGTT GCTGCTCGTTCCAC-3'), 9648F (5'-AGTTGTGTCAGTGGCC TCGTTC-3') and 10403R (5'-TAAAGGACGCGGAGCTTAGC TG-3'). Amplicons were subjected to Sanger sequencing and nucleotide assembly with BioEdit version 7.2.5 (bioedit.software.informer.com).

Phylogenetic analysis. To establish genetic relationship among the CHIKV strains, we initially analyzed the nucleotide sequences using BLAST (http://blast.ncbi.nlm.nih.gov). Sequences were aligned using the BioEdit program v7.2.5 (http://www.mbio.ncsu.edu/bioedit/bioedit. html). Phylogenetic analysis was performed by using the maximum likelihood method implemented in MEGA5. ${ }^{23}$ Consistency of branching was tested by bootstrap analysis of 1,000 resamplings.
CHIKV antibody assay. Serum samples were tested for CHIKV IgM and IgG by using a commercial immunochromatographic assay (STANDARD Q Chikungunya IgM/lgG, SD Biosensors, Suwon, Korea). Results were interpreted by three independent laboratory technicians, at least two of whom must agree prior to result acceptance.

\section{RESULTS}

CHIKV RNA was identified in 17\% (21/127) of the samples (mean Ct value 22.2, range 17.64-32.01). CHIKV RNA was observed in $18 \%(7 / 38)$ males and $16 \%$ (14/89) females. Over half of the CHIKV-infected individuals were between the ages of 20 and 60 years (57\%), followed by older adults (19\%), teenagers (19\%), and children (5\%). Collectively, patients reported experiencing fever (80\%), rash (52\%), myalgia $(72 \%)$, and arthralgia (83\%). Six of the 18 pools of $A$. aegypti and one of the three $A$. albopictus mosquitoes collected in the vicinity of the homes where patients reside also tested positive for CHIKV RNA.

We also examined immunological markers of CHIKV among the samples and found that $14 \%(18 / 127)$ of the samples were simultaneously positive for both IgM and IgG. Additionally, 9.4\% (12/127) and 7.9\% (10/127) demonstrated only CHIKV IgM or IgG, respectively. Acute infection possessing both CHIKV RNA and IgM was identified in one sample. If we defined recent CHIKV by the presence of either CHIKV RNA, $39 \%(50 / 127)$ of the samples would fit under this criteria.

To further characterize the genetic relationships between the Satun CHIKV strains in this study with previously strains, 15 CHIKV-positive samples with high viral loads were subjected to sequence analysis. The region encompassing 485 nucleotides obtained from the Satun strains was identical except $\mathrm{C} / \mathrm{T}$ differences at position 507, which was a silent mutation at amino acid residue 169 in the E1 protein. Next, their nucleotide sequences were compared with the previous CHIKV strains identified in Thailand and in other countries. All grouped with reference strains of the Indian Ocean clade within the ECSA lineage (Figure 1). In addition to similarities with the previous Thai strains identified over the past 10 years, the Satun strains were also genetically similar to the more recent strains identified from CHIKV outbreaks in 2016 in India, Pakistan, and Australia, and in 2017 in Bangladesh, Italy, and China.

The deduced amino acid sequence, which represents residues 96 to 256 of E1, showed that all Satun strains also have alanine at residue position 226 (E1:A226). In contrast to the Ross strain and previous CHIKV strains identified in Thailand in the past 10 years, however, the Satun strains have E1:K211E (Figure 2). Analysis of the complete E2 genes on a subset of the samples in this study, including four patient samples and three Aedes aegypti mosquitoes, showed 100\% deduced amino acid sequence identity. Comparison with the LR2006 reference strain (GenBank accession number DQ443544) identified two residue changes (G205S and V264A). In summary, the Satun strains appear to be different from previously circulating CHIKV in Thailand.

\section{DISCUSSION}

In this study, $39 \%$ of the individuals living in a community in Satun province who reported experiencing acute febrile illness during the 2018 rainy season demonstrated the presence of 


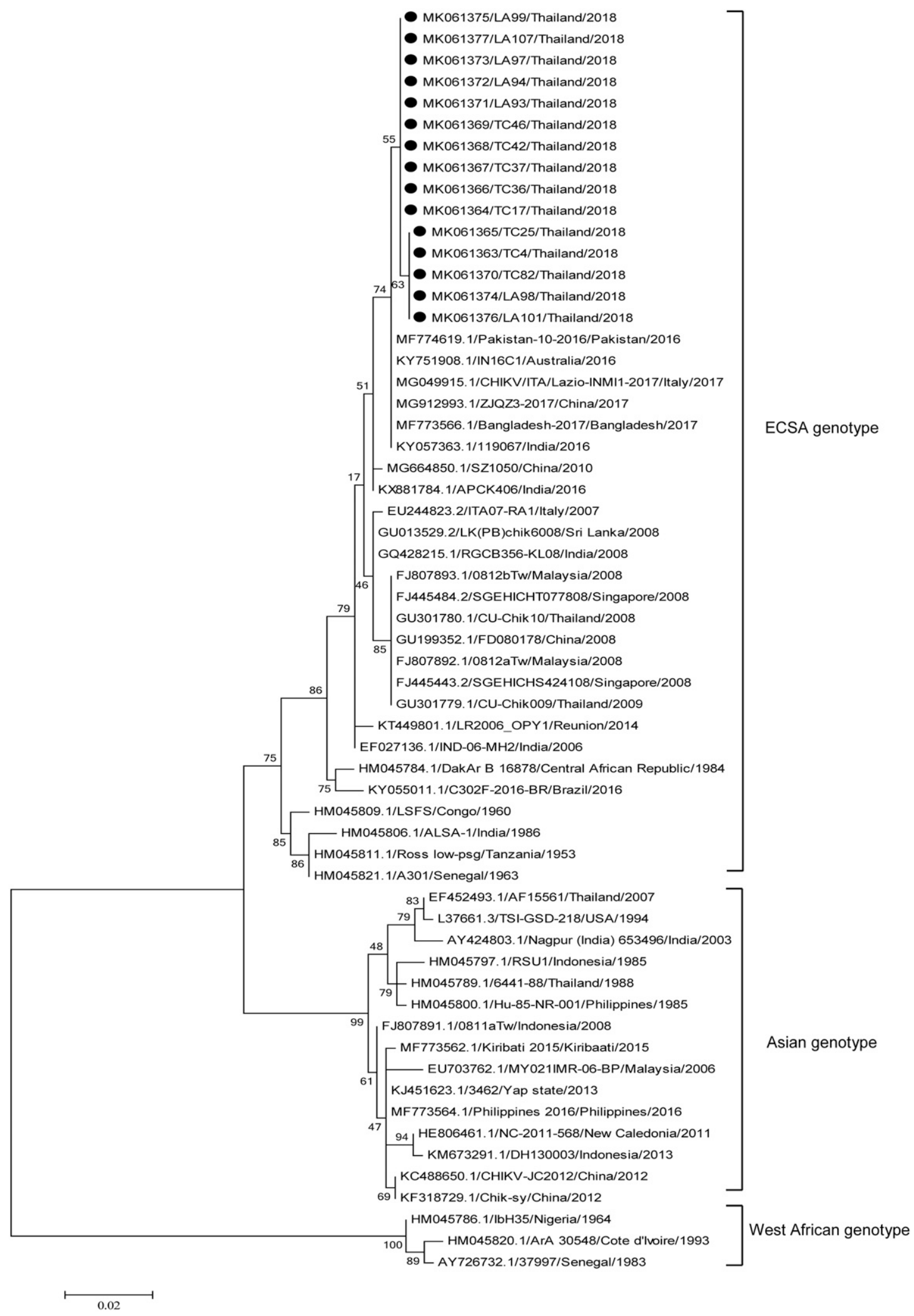

FIGURE 1. Phylogenetic analysis of the partial E1 gene sequences of chikungunya virus. Strains identified in this study were compared with the global CHIKV sequences available from GenBank. Trees were generated using the maximum likelihood method implemented in MEGA5. Bootstrap values are indicated at the branch nodes. Strains are identified using GenBank accession number/strain/country/year of isolation. Scale bar indicates the genetic distance in nucleotide substitutions per site. Black circles represent CHIKV strains from this study (GenBank accession numbers MK061373 to MK061376). 
(96) $96 \quad 110 \quad 120 \quad 130$

140

150

160

170 180

HM045811.1/Ross/1953 (96) CDAENTQLSEAHVEKSESCKTEFASAYRAHTASASAKLRVLYQGNNITVTAYANGDHAVTVKDAKFIVGPMSSAWTPFDNKIVVY KT449801.1/LR2006/2014 (96) CDAENTQLSEAHVEKSESCKTEFASAYRAHTASASAKLRVLYQGNNITVTAYANGDHAVTVKDAKFIVGPMSSAWTPFDNKIVVY GU301780.1/CU/2008 (1) CDAENTQLSEAHVEKSESCKTEFASAYRAHTASASAKLRVLYQGNNITVTAYANGDHAVTVKDAKFIVGPMSSAWTPFDNKIVVY KJ579184.1/BK46/2013 (1) CDAENTQLSEAHVEKSESCKTEFASAYRAHTASASAKLRVLYQGNNI TVTAYANGDHAVTVKDAKFIVGPMSSAWTPFDNKIVVY Satun_2018 (1) CDAENTQLSEAHVEKSESCKTEFASAYRAHTASASAKLRVLYQGNNITVTAYANGDHAVTVKDAKFIVGPMSSAWTPFDNKIVVY

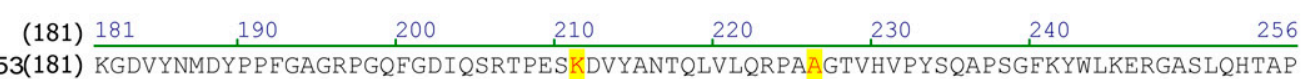

HM045811.1/Ross/1953(181) KGDVYNMDYPPFGAGRPGQFGDIQSRTPESKDVYANTQLVLQRPAAGTVHVPYSQAPSGFKYWLKERGASLQHTAP

KT449801.1/LR2006/2014(181) KGDVYNDYP
GU301780.1/CU/2008 (86) KGDVYNMPPPFGAGRPGQFGDIQSRTPESKDVYANTQLVLQRPAVGTVHVPYSQAPSGFKYWLKERGASLQHTAP

KJ579184.1/BK46/2013 (86) KGDVYNMDYPPFGAGRPGQFGDIQSRTPESKDVYANTQLVLQRPAVGTVHVPYSQAPSGFKYWLKERGASLQHTAP Satun_2018 (86) KGDVYNMDYPPFGAGRPGQFGDIQSRTPESEDVYANTQLVLQRPAAGTVHVPYSQAPSGFKYWLKERGASLQHTAP

FIGURE 2. Alignment of the amino acid sequence corresponding to residues 96 to 256 . The deduced residues of a representative Satun strain was compared with the reference strain Ross, LR2006, and representative chikungunya virus strains identified during previous outbreaks in Thailand. This figure appears in color at www.ajtmh.org.

either CHIKV RNA or IgM around the time or shortly after their illness, which suggests a CHIKV outbreak. Approximately, one in six individuals tested in this study had CHIKV viremia at the time their samples were tested. This result is consistent with the epidemiological data from the Bureau of Epidemiology in the Department of Disease Control, which recorded an upsurge of suspected and confirmed CHIKV in the Satun region including the neighboring provinces of Songkhla, Pattani, and Narathiwat, around the same time. The latter province previously experienced a large CHIKV outbreak in 2008-2009. ${ }^{20,21}$ In 2018, national statistics indicated that approximately 3,580 reported cases of CHIKV infection with an estimated infection rate of 5.39 people per 100,000 people represents more than 100 -fold increase in $\mathrm{CHIKV}$ over the past 5 years. For southern Thailand alone, the infection rate was 37.57 people per 100,000 , which may be an underestimate given the historically incomplete reporting by local health officials. The reason for this increase may be attributed to the underrecognition of chikungunya disease, misdiagnosis as dengue due to the close clinical resemblance, or an actual bona fide increase in chikungunya prevalence. No cases of CHIKV fever had previously been reported in Satun province before 2018, and only sporadic imported cases have been seen in the nearby southern provinces where tourism dominates the local economy. European travelers to southern Thai provinces of Phuket and Krabi have been infected with CHIKV in late $2018 .^{24,25}$ Viral transmission is aided by the mobility of people and mosquitoes as a result of human migration and air travel. Furthermore, the sparsely populated Satun, which is heavily forested and mountainous, would provide an ideal habitat for Aedes spp. mosquitoes especially during the rainy season. This could be another explanation for why the chikungunya outbreak was discovered in this area. Such occurrence has not been extensively documented since the 2008-2009 CHIKV outbreak in the Malay Peninsula including southern Thailand. ${ }^{14,20,21}$ Although preexisting immunity attained from prior CHIKV exposure should have attenuated reinfections, we believe that the increased prevalence of CHIKV in 2018 may have infected those residing in areas minimally affected by the 2008-2009 outbreak. Although waning immunity in the previously infected may have rendered some individuals susceptible to reinfection, it is also possible that the differences in amino acid residues in this most recent CHIKV strain influence viral fitness and infectivity as compared with the 2008-2009 ECSA strains. Even though ECSA lineage has predominated in Thailand for the past decade, the CHIKV strains from this study possessed E1:K211E and E1:A226, which are different from past CHIKV strains identified in Thailand. Both of these residues are located at the receptor binding interface and allowed increased infectious titers in $A$. aegypti. ${ }^{9,26}$ Of the two changes on the $E 2$ gene from the strains identified in this study, V264A has previously been implicated in the enhancement of fitness in A. aegypti. ${ }^{9}$ Taken together, this suggests that the $2018 \mathrm{CHIKV}$ may not have been endemic to Thailand nor had evolved from earlier circulating strains. These signature residues have been reported in CHIKV outbreaks including in Pakistan, India, Bangladesh, and China. ECSA remains the predominant CHIKV lineage in the neighboring Malaysia, but Indonesia has reported the continued predominance of the Asian lineage with sporadic ECSA strains detected near the border with Malaysia. ${ }^{27}$

This study was limited by the small number of samples tested for CHIKV. We did not attempt to identify other arbovirus infections including dengue and Zika virus, which may have been cocirculating. It has been reported that $\mathrm{CHIKV}$ in serum is detectable by RT-PCR up to day 6 after the onset of fever; therefore, individuals who tested CHIKV-negative may have had false-negative result as the acute phase had passed. ${ }^{28}$ In summary, data from this study will be important to document $\mathrm{CHIKV}$ surveillance effort and track CHIKV activity in this region.

Received April 20, 2021. Accepted for publication June 4, 2021.

Published online August 2, 2021.

Acknowledgments: We are grateful to the staff of Langu hospital and The Office of Disease Prevention and Control 12 in Songkhla province for their tireless assistance in sample collection.

Financial support: This work was supported by The Thailand Research Fund (MRG5980203) and by the Faculty of Medical Technology, Prince of Songkla University.

Authors' addresses: Natthaphon Nanakorn and Theerakamol Pengsakul, Faculty of Medical Technology, Prince of Songkla University, Songkhla, Thailand, E-mails: natthaphon.s@psu.ac.th and theerakamol.p@psu.ac.th. Kannika Bunrod, Langu Hospital, Satun, Thailand, E-mail: langulab99@gmail.com. Suwich Thammapalo and Pathomporn Prikchoo, Office of Disease Prevention and Control 12, Songkhla, Thailand, E-mails: suwich.t@ddc.mail.go.th and pathomporn.p@ddc.mail.go.th. Sompong Vongpunsawad and Yong Poovorawan, Center of Excellence in Clinical Virology, Faculty of Medicine, Chulalongkorn University, Bangkok, Thailand, E-mails: sompong.vo@chula.ac.th and yong.p@chula.ac.th. 


\section{REFERENCES}

1. Lumsden WH, 1955. An epidemic of virus disease in Southern Province, Tanganyika Territory, in 1952-53. II. General description and epidemiology. Trans R Soc Trop Med Hyg 49: 33-57.

2. Burt FJ, Rolph MS, Rulli NE, Mahalingam S, Heise MT, 2012. Chikungunya: a re-emerging virus. Lancet 379: 662-671.

3. Robinson MC, 1955. An epidemic of virus disease in southern Province, Tanganyika Territory, in 1952-53. I. Clinical features. Trans $R$ Soc Trop Med Hyg 49: 28-32.

4. Schwartz O, Albert ML, 2010. Biology and pathogenesis of Chikungunya virus. Nat Rev Microbiol 8: 491-500.

5. Sun S et al., 2013. Structural analyses at pseudo atomic resolution of Chikungunya virus and antibodies show mechanisms of neutralization. eLife 2: e00435.

6. Li L, Jose J, Xiang Y, Kuhn RJ, Rossmann MG, 2010. Structural changes of envelope proteins during alphavirus fusion. Nature 468: 705-708.

7. Volk SM et al., 2010. Genome-scale phylogenetic analyses of chikungunya virus reveal independent emergences of recent epidemics and various evolutionary rates. J Virol 84: 6497-6504.

8. Tsetsarkin KA, Vanlandingham DL, McGee CE, Higgs S, 2007. A single mutation in chikungunya virus affects vector specificity and epidemic potential. PLoS Pathog 3: e201.

9. Agarwal A, Sharma AK, Sukumaran D, Parida M, Dash PK, 2016. Two novel epistatic mutations (E1:K211E and E2:V264A) in structural proteins of Chikungunya virus enhance fitness in Aedes aegypti. Virology 497: 59-68.

10. AbuBakar S, Sam IC, Wong PF, MatRahim N, Hooi PS, Roslan N, 2007. Reemergence of endemic Chikungunya, Malaysia. Emerg Infect Dis 13: 147-149.

11. Centers for Disease C, 2012. Prevention. Chikungunya outbreak-Cambodia, February-March 2012. MMWR Morb Mortal Wkly Rep 61: 737-740.

12. Duong V et al., 2012. Reemergence of Chikungunya virus in Cambodia. Emerg Infect Dis 18: 2066-2069.

13. Laras $\mathrm{K}$ et al., 2005. Tracking the re-emergence of epidemic Chikungunya virus in Indonesia. Trans $R$ Soc Trop Med Hyg 99: 128-141.

14. Leo YS, Chow AL, Tan LK, Lye DC, Lin L, Ng LC, 2009. Chikungunya outbreak, Singapore, 2008. Emerg Infect Dis 15: 836-837.

15. Liew C, Yung CF, 2012. First detection of Chikungunya infection and transmission in Brunei Darussalam. Singapore Med J 53: e66-e68.
16. Salje $\mathrm{H}$ et al., 2016. Reconstruction of 60 years of Chikungunya epidemiology in the Philippines demonstrates episodic and focal transmission. J Infect Dis 213: 604-610.

17. Sasmono RT et al., 2017. Chikungunya detection during dengue outbreak in Sumatra, Indonesia: clinical manifestations and virological profile. Am J Trop Med Hyg 97: 1393-1398.

18. Tun MM et al., 2014. Detection of east/central/south African genotype of Chikungunya virus in Myanmar, 2010. Emerg Infect Dis 20: 1378-1381.

19. Hammon WM, Rudnick A, Sather GE, 1960. Viruses associated with epidemic hemorrhagic fevers of the Philippines and Thailand. Science 131: 1102-1103.

20. Pongsiri P, Auksornkitti V, Theamboonlers A, Luplertlop N, Rianthavorn P, Poovorawan Y, 2010. Entire genome characterization of Chikungunya virus from the 2008-2009 outbreaks in Thailand. Trop Biomed 27: 167-176.

21. Rianthavorn $P$, Prianantathavorn K, Wuttirattanakowit N, Theamboonlers A, Poovorawan Y, 2010. An outbreak of chikungunya in southern Thailand from 2008 to 2009 caused by African strains with A226V mutation. Int J Infect Dis 14 (Supp/ 3): e161-e165

22. Lanciotti RS et al., 2007. Chikungunya virus in US travelers returning from India, 2006. Emerg Infect Dis 13: 764-767.

23. Tamura K, Peterson D, Peterson N, Stecher G, Nei M, Kumar S, 2011. MEGA5: molecular evolutionary genetics analysis using maximum likelihood, evolutionary distance, and maximum parsimony methods. Mol Biol Evol 28: 2731-2739.

24. Javelle $E$ et al., 2019. Increased risk of chikungunya infection in travellers to Thailand during ongoing outbreak in tourist areas: cases imported to Europe and the Middle East, early 2019. Euro Surveill 24: 1900146.

25. Kantele A, 2019. Travellers as sentinels of Chikungunya epidemics: a family cluster among Finnish travellers to Koh Lanta, Thailand, January 2019. Euro Surveill 24: 1900162.

26. Basore $\mathrm{K}$ et al., 2019. Cryo-EM structure of Chikungunya virus in complex with the Mxra8 receptor. Cell 177: 1725-37 e16.

27. Maha MS, Susilarini NK, Hariastuti NI, 2015. Subangkit. Chikungunya virus mutation, Indonesia, 2011. Emerg Infect Dis 21: 379-381.

28. Chusri S et al., 2014. Kinetics of Chikungunya infections during an outbreak in Southern Thailand, 2008-2009. Am J Trop Med Hyg 90: $410-417$. 